\title{
Dismorfia muscular: A busca pelo corpo hiper musculoso
}

\author{
Muscle dysmorphia: A quest for the hyper muscular body
}

\author{
A.P. Azevedo, A.C. Ferreira, P.P. Da Silva, I.O. Caminha, C.M. Freitas
}

ARTIGO ORIGINAL | ORIGINAL ARTICLE

\begin{abstract}
A dismorfia muscular tem sido identificada tanto em homens quanto em mulheres, provocando alterações da perceção da autoimagem e prejuízos socioculturais, e na saúde e bem-estar dos indivíduos. Nesse sentido, o objetivo deste estudo é analisar os aspetos socioculturais, psicológicos e o uso de recursos ergogênicos relacionados à dismorfia muscular, bem como, identificar os riscos promovidos pelo transtorno. Participaram 20 indivíduos, inscritos na rede mundial de computadores. Utilizou-se um questionário para coleta de dados e a análise documental para interpretação das respostas. Os resultados demonstram que as preocupações com a imagem corporal geram insegurança social, baixa autoestima e sentimentos de inferioridade, que seriam resolvidos se a pessoa tivesse corpos belos e fortes. A dismorfia muscular pode aumentar o risco de uso dos esteroides anabolizantes e o uso indiscriminado de suplementos alimentares. Conclui-se que a dismorfia muscular causa sofrimentos e prejuízos psicológicos, socioculturais e, desse modo, compromete a saúde das pessoas. Palavras-chave: vigorexia, transtorno dismórfico muscular, recurso ergogênico, imagem corporal
\end{abstract}

ABSTRACT

Muscle dysmorphia has been identified both in men and women, causing changes in the perception of self image and sociocultural losses and in the health and well being. In this sense, the purpose of this study was to examine the sociocultural and psychological aspects and the use of ergogenic resources related to muscle dysmorphia as well to identify the risks promoted by the disorder. The participants included 20 individuals enrolled by the Internet. We used a questionnaire to gather data and document analysis to interpret the responses. The results showed that concerns about body image generate insecurity, low self-esteem and feelings of inferiority, which would be solved if people had beautiful and strong bodies. Muscle dysmorphia can increase the risk of using anabolic steroids and indiscriminate use of dietary supplements. We conclude that muscle dysmorphia causes suffering, psychological and sociocultural damage, and thus compromises the health of people.

Keywords: vigorexia, muscle dysmorphic disorder, muscle dysmorphia, ergogenic aid, body image

Submetido: 28.06.2010 | Aceite: 06.03.2011

Andréa Pires Azevedo e Iraquitan de Oliveira Caminha. Laboratório de Estudo e Pesquisa em Lazer, Esporte, Corpo e Sociedade; Programa Associado de Pós-graduação em Educação Física UPE/UFPB, Paraíba, Brasil.

Alan Carvalho Ferreira. Departamento de Nutrição, Universidade Federal da Paraíba, Brasil.

Priscila Pinto Da Silva e Clara Monteiro de Freitas. Laboratório de Práticas Esportivas e Lazer; Programa Associado de Pós-graduação em Educação Física UPE/UFPB, Paraíba, Brasil.

Endereço para correspondência: Andréa Pires Azevedo, SQN 407 - Bloco E, apto. 212, Asa Norte, CEP: 70855-050 Brasília-DF, Brasil

E-mail: andrea.mpaf@hotmail.com 
O transtorno dismórfico corporal tem por característica principal alterações na perceção da autoimagem, preocupações irracionais de possíveis imperfeições na aparência, de modo acentuadamente excessivo e desproporcional à realidade, gerando importantes prejuízos no funcionamento pessoal, familiar, social e profissional (Fairburn, 1994; Torres, Ferrão, \& Miguel, 2005).

Na década de 1990, a preocupação com a imagem corporal era eminentemente feminina, relacionada à anorexia e à bulimia. Atualmente, estas preocupações corporais afetam ambos os sexos, e de forma crescente os homens, sendo denominadas de dismorfia muscular (DM) ou vigorexia (Hildelbrandt, Langenbu-cher, \& Schlundt, 2004).

A dismorfia muscular é um transtorno recentemente descrito e ainda não consta nos manuais de diagnóstico em psiquiatria e seu quadro clínico ainda não está devidamente definido. Também são escassos estudos epidemiológicos sobre o transtorno, sendo a maior parte dos dados científicos obtidos a partir de atletas ou fisiculturistas, prejudicando generalizações sobre a prevalência ou incidência desse quadro. Alguns autores sugerem que o transtorno origina-se por fatores socioculturais (Pope Jr, Gruber, Choi, Olivardia, \& Phillips, 1997). Pesquisadores argumentaram que a DM comummente se manifesta quando os indivíduos recebem pressões sociais para alcançar um determinado padrão corporal (Cohane \& Pope Jr, 2001; Olivardia, 2001; Pope Jr, Phillips, \& Olivardia, 2000).

Outros pensam que a DM decorre de uma baixa autoestima, insatisfação e distorção da aparência do corpo, associadas a fatores biológicos e sociais (Lantz, Rhea, \& Mayhew, 2001). Grieve (2007) propôs que as variáveis mais importantes relacionadas à dismorfia muscular são a distorção da perceção da imagem corporal, a insatisfação com o corpo, e a construção de uma imagem corporal ideal internalizada. Essas três variáveis, juntamente com perfeccionismo, afeto negativo, baixa autoestima e pressão da mídia são pensadas como a base das condições necessárias para o desenvolvimento da dismorfia muscular.

A identificação precoce da dismorfia muscular minimiza o uso de drogas que podem ser nocivas ao corpo e a mente, por exemplo, os esteroides anabólicos androgénicos (EAA), geralmente administrados para se obter rapidamente os resultados desejados ou fantasiados, como um corpo perfeitamente hipertrofiado e forte. Na maioria dos casos, tal comportamento é fruto da busca pela aceitação social e, decorrente da pressão exercida pela mídia, na qual o narcisismo se manifesta muito atuante (Rohman, 2009).

Neste contexto, fica clara a íntima relação entre a busca pelo corpo musculoso e a ausência de limites para atingir tal objetivo, conduzindo pessoas à procura de um corpo exageradamente hipertrofiado. Sujeitos que praticam o treinamento de força exaustivamente não apenas em busca do bem-estar, mas principalmente objetivando corpos progressivamente musculosos, são sérios candidatos ao diagnóstico de DM. Normalmente estes indivíduos estão dispostos a manter uma dieta principalmente hiperproteica e hipolipídica, a usar fármacos e praticar o treinamento de força de maneira demasiadamente intensa para conseguir o objetivo de um corpo perfeito, que jamais é alcançado.

Desta forma, o presente estudo tem o objetivo de analisar os aspetos socioculturais, psicológicos e o uso de recursos ergogênicos relacionados à dismorfia muscular, identificando os riscos à saúde promovidos por esse transtorno. Espera-se que o trabalho possa contribuir para melhor entendimento das práticas de hiperinvestimento no corpo, vistas na sociedade contemporânea consumista, principalmente no que diz respeito à compulsão pela aquisição de um corpo musculoso, tendo como referência temática as noções de dismorfia muscular.

\section{MÉTODO}

Foi realizado um estudo transversal exploratório, de abordagem quali-quantitativa. 


\section{Amostra}

Foram credenciados para o estudo 20 indivíduos, de ambos os sexos, com idade a partir de 18 anos, praticantes de musculação e inscritos em comunidades específicas sobre dismorfia muscular, na rede mundial de computadores. Estes foram selecionados por meio da mídia eletrônica, considerando sua disposição de participar da pesquisa via correio eletrônico. Importa salientar que para resguardar a identidade dos participantes, atribuímos nomes fictícios de personagens que remetem a imagens de corpos musculosos.

Foram consideradas as orientações sugeridas pela Resolução no 196/96 do Conselho Nacional de Saúde / MS, visando assegurar os direitos e deveres que dizem respeito à comunidade cientifica, ao(s) sujeito(s) e ao Estado. A metodologia deste estudo foi aprovada pelo Comitê de Ética em Pesquisa em Seres Humanos do Hospital Universitário Lauro Wanderley da Universidade Federal da Paraíba - UFPB sob o Protocolo n ${ }^{\circ} 378 / 2009$.

\section{Instrumentos e Procedimentos}

$\mathrm{O}$ instrumento para coleta de dados foi um questionário de identificação e caracterização dos sujeitos, estruturado e fundamentado a partir de artigos científicos da literatura (Azevedo, 2008; Dawes \& Mankin, 2004). Foram contempladas variáveis objetivas relacionadas ao perfil e caracterização socioeconômica do grupo (sexo, escolaridade, renda familiar, estado civil, tempo de prática da musculação, frequência de treino, tempo gasto em cada sessão) e subjetivas (idade, tempo de uso e tipo/nome relativos aos suplementos alimentares e esteroides anabolizantes, tipo de dieta e incômodos quanto à aparência física). Por fim, os dados acerca da rotina alimentar e imagem corporal dos sujeitos foram analisados. O questionário foi estruturado de forma a ser respondido voluntária e anonimamente.

A abordagem quali-quantitativa da pesquisa não admitiu uma testagem dos instrumentos, própria da investigação convencional, como afirma Freitas (2000). No entanto, houve o cuidado de aplicar 10 questionários-piloto, composto por 15 itens distribuídos nas dimensões supracitadas objetivando optar por questões que se revelaram imprescindíveis diante do fenômeno, no sentido de potencializar e apreender a realidade estudada.

\section{Procedimentos para Coleta de Dados}

O contato com os indivíduos incluídos na pesquisa foi realizado nos meses de novembro e dezembro de 2009. Inicialmente foi criado um perfil pelos pesquisadores em 52 comunidades relacionadas à "Vigorexia", "Viciados em Academia", "Vigorexia Síndrome de Adônis", "Vigorexia®", "Vigorexia. Doença ou não?", "Perdi um amigo para VIGOREXIA!", "Feio de tão grande é o @\#\$\%“\&o”, "Eu tenho VIGOREXIA e DAí?", "Vigorexia, um estilo de vida.", "Fisiculturismo", etc., dos sites de relacionamentos (ORKUT e BLOGS), totalizando o contato com 125003 indivíduos pertencentes às comunidades. Nestas, foram postadas informações pertinentes ao estudo, bem como foi divulgado o endereço eletrônico da pesquisadora principal para os internautas que apresentassem interesse em receber e preencher o questionário.

Os sujeitos do estudo estavam cientes de sua participação voluntária e de não haver nenhuma consequência pela sua não-participação. Foi ressaltada pela pesquisadora a importância da fidedignidade dos dados fornecidos. Todos os participantes eram membros de pelo menos uma comunidade do site de relacionamentos ORKUT ${ }^{\circledR}$.

\section{Técnicas de Análise dos Dados}

Após o recebimento dos questionários, foi realizada uma análise quantitativa dos dados obtidos por meio de estatística descritiva, como frequência, percentual, média e desvio padrão. Para decompor as questões subjetivas foi utilizada a técnica de análise de conteúdo a fim de desvelar e compreender os fenômenos que envolvem a dismorfia muscular. A organização da análise de conteúdo envolveu três fases: pré-análise, exploração do material e 
análise de interpretação dos resultados (Bardin, 2002). A pré-análise dos registros foi realizada por meio da leitura flutuante, ou seja, este foi o momento em que houve organização do material, formulação das questões norteadoras e elaboração de indicadores que fundamentaram a interpretação final. Durante a fase de exploração do material houve a tomada das decisões adotadas na pré-análise. Foi o momento da codificação, em que os dados brutos foram categorizados de forma organizada e agregados em unidades, as quais permitiram uma descrição das características relacionadas aos conteúdos. Nesse sentido são nomeadas as características sociodemográficas, as práticas do treinamento de força, o uso de esteroides anabólicos androgénicos, os aspetos psicossociais, de imagem corporal e nutricional.

\section{APRESENTAÇÃO E DISCUSSÃO DOS RESULTADOS}

As reflexões empreendidas neste estudo foram focadas na compreensão dos aspetos sociopsicoculturais, e do uso de recursos ergogênicos, definida pelos atores sociais que apresentam compulsão pela prática do treinamento de força. Desta maneira, foi possível perceber, ao longo do trabalho, que não é aceitável uma extensão desta temática sem estabelecer uma relação indissociável entre os contextos sociopsicoculturais e biológico.

\section{Dados Sociodemográficos}

No que concerne aos dados sociodemográficos, dos 20 atores sociais participantes do presente estudo, constatou-se que todos eram solteiros e $85 \%$ eram do sexo masculino. A idade apresentou variação entre 18 e 24 anos (19.6 \pm 1.98 anos). Em relação à renda mensal familiar, $50 \%$ dos sujeitos relataram ter renda igual ou superior a sete salários mínimos. Importa salientar que atualmente o valor do salário mínimo é de $\mathrm{R} \$ 510.00$ ou cerca de $€$ 214.00. A relação entre a dismorfia muscular e o sexo masculino pode ser atribuída às características do transtorno, uma vez que as alterações na perceção da imagem corporal e a con- sequente compulsão pelo treinamento de força conduzem a uma progressiva hipertrofia muscular. Esta, conforme afirmam Pope Jr et al. (1997) e Olivardia (2001) é um desejo principalmente de indivíduos do sexo masculino. No entanto, mulheres cada vez mais estão demonstrando este anseio pela estética musculosa, tendo em vista a popularização das modelos fisiculturistas concomitantemente aos apelos da mídia. Em 2000, Olivardia, Pope Jr e Hudson, encontraram maior incidência de transtornos de humor, transtornos de ansiedade e transtornos alimentares em homens com dismorfia muscular do que em sujeitos sem este transtorno. Os pesquisadores também evidenciaram que entre $5 \%$ e $10 \%$ dos levantadores de peso e $9 \%$ dos pacientes com transtorno dismórfico corporal possuíam dismorfia muscular.

No que concerne à faixa etária, a investigação realizada por Vieira, Rocha e Ferrarezzi (2010) corrobora com os resultados do presente estudo, apresentando idade média de 24 anos. Fato este que pode ser explicado pelo início da dependência pelo treinamento de força na adolescência em função de pressões sociais advindas principalmente da família e amigos, e o agravamento desta dependência na fase adulta podem ocorrer, segundo Swami e Tovvé (2007) e Vieira et al. (2010), em consequência às preocupações e exigências sociais referentes à imagem corporal na tentativa de anular os efeitos ocasionados pela idade cronológica, pelos hábitos de vida adquiridos sobre a autoimagem, e pelas exigências da mídia em busca da aceitação social e realização.

De maneira geral é nítida a predominância de adultos jovens na prática do treinamento de força. Em Curitiba, $61.2 \%$ dos frequentadores de academia tinham entre 20 e 29 anos (Reis, Manzoni, \& Simonard-Loureiro, 2006). Em Campinas $59.6 \%$ dos praticantes de treinamento de força pesquisados tinham entre 17 e 28 anos (Lollo \& Tavares, 2004). Assim, os resultados da presente pesquisa corroboram com os achados científicos que demonstram associação entre o período inicial da dismorfia 
muscular - no final da adolescência - e seu desenvolvimento, período no qual naturalmente as pessoas apresentam maior insatisfação com o próprio corpo e se submetem exageradamente à prática do treinamento de força, sem levar em consideração os riscos à saúde decorrente da busca de se atingir um corpo musculoso a qualquer preço. $\mathrm{Na}$ adolescência, existem cobranças, principalmente socioculturais, para que os meninos fiquem fortes e musculosos. A esse respeito, Arraz (2010, p. 8) cita "...Stallone, Schwarze-negger, Van Damme, He-Man, Super Homem, Conan ,... difíil encontrar um adolescente que não tenha, ao menos uma vez, se inspirado num desses ícones da virilidade masculina, com músculos enormes e corpo dividido. Estimulados progressivamente pela mídia, que associa a idéia do corpo perfeito ao sucesso e felicidade, e pela enorme difusão de academias de ginástica nos anos 90, os jovens 'marombeiros' foram em busca do sonho de ter o poder e o respeito encarnados numa silhueta musculosa".

Ainda, de acordo com Beiras, Lodetti, Cabral, Toneli e Raimundo (2007) as histórias em quadrinhos de super-heróis ganham vida na imaginação dos leitores, estabelecendo fortes ligações com seu cotidiano. Assim, estas caracterizações remetem a tendências distintas de representação dos corpos, (re)produzindo normas sociais e valores estéticos sobre a corporeidade, principalmente no universo masculino, cuja imagem do corpo está associada ao modelo de virilidade instituído socialmente.

No que concerne ao nível de escolaridade, a maioria dos participantes estava cursando ou havia cursado o ensino superior (50\%), o que demonstra um elevado grau de instrução destes praticantes, supostamente com maiores possibilidades de acesso às informações, além do potencial para o desenvolvimento de análises críticas. Além disso, a maior prevalência no ensino superior é condizente com a faixa de idade mais prevalente entre os atores. Pesquisas realizadas por Pope Jr, Katz e Hudson (1993) e Pope Jr et al. (1997, 2000) identificaram que sujeitos com altos níveis de escolaridade sofriam de dismorfia muscular, apresentando necessidade compulsiva de praticar o treinamento de força. Dentre eles, graduados em diversas profissões, inclusive na área de saúde como educadores físicos e médicos e ainda, sujeitos com título de Ph.D. (Philosophy Doctor).

Neste sentido, é importante apresentar estudos realizados com praticantes de treinamento de força no Brasil. Nas academias de Porto Alegre (RS) foram encontrados resultados semelhantes, com predominância de praticantes de treinamento de força cursando o ensino superior, onde $36 \%$ possuíam ensino superior completo e $40 \%$ ensino superior incompleto (Silva et al., 2007). Achados de Frizon, Macedo e Yonamine (2005) em pesquisa também realizada no RS, apontou que estudantes universitários praticam o treinamento de força e fazem uso de substâncias injetáveis, sendo motivados principalmente pela estética. Santos e Santos (2002) na cidade de Vitória no Estado do Espírito Santo, observaram que $76 \%$ dos frequentadores de academias estavam cursando o ensino superior e, em São Paulo, $69.9 \%$ dos entrevistados em academias de ginástica possuíam curso superior (Pereira, Lajolo, \& Hirschbruch, 2003), o que evidencia um grau de escolaridade similar ao encontrado no presente estudo.

No tocante a variável renda familiar, $50 \%$ dos sujeitos referiram aquela acima de sete salários mínimos ( $\$ 3570.00$ ou cerca de $€$ 1516.00). Estes achados nos permitem inferir e direcionam para uma tendência de que os indivíduos com dismorfia muscular possuem encargos financeiros aproximadamente três vezes mais da média salarial dos brasileiros que, de acordo com a pesquisa realizada em 2009 pela instituição financeira Cetelem em parceria com o instituto de pesquisas Ipsos, é de $R \$ 1201.00$ ou cerca de $€ 510.00$. Assim, a renda mensal dos vigoréxicos potencializam a aquisição e utilização de recursos ergogênicos como anabolizantes e suplementos alimentares, que possuem custo elevado.

Investigação de Azevedo (2008) encontrou resultados análogos ao do atual estudo quando a prevalência da renda familiar mensal de indi- 
víduos com dismorfia muscular foi igual ou maior do que sete salários mínimos. Resultado diferente ao do presente estudo foi encontrado por Silva Júnior, Souza, Silva, Oliveira e Souza (2008), na cidade do Rio de Janeiro, onde apenas $4.3 \%$ dos pesquisados relataram renda entre sete e nove salários mínimos, $13.0 \%$ declaram possuir renda maior que 10 salários mínimos e $43.5 \%$ entre um e três. Estes achados demonstram que o treinamento de força é um exercício físico difundido e acessível para indivíduos e comunidades de todas as classes sociais.

\section{Treinamento de Força}

Em relação aos aspetos da prática do treinamento de força, tantos os atores do sexo masculino, quanto feminino, apresentaram resultados análogos. Verificou-se que $50 \%$ da amostra treina há mais de três anos (3.3 \pm 1.89 horas), com frequência semanal de cinco vezes $(5.3 \pm 0.66$ vezes/semana), usando mais de uma hora e meia de treino $(1.55 \pm 0.83$ horas) por sessão. Esses resultados foram similares aos verificados em diversas cidades do país (Araújo \& Soares, 1999; Lollo \& Tavares, 2004; Silva et al., 2007; Silva Júnior et al., 2003), nos quais um número expressivo dos praticantes de treinamento de força o realizam cinco ou mais vezes por semana, com duração de duas horas para cada sessão de treino.

De acordo com Pereira, Doimo e Kowalski (2009), atualmente algumas práticas corporais tais como a compulsão pelo treinamento de força, vem sendo justificadas pelo desejo de se ter um corpo ideal. A aparência física parece ser forte elemento nas relações sociais, pois a ela são atribuídos significados e, ao almejar esses modelos corpóreos, o resultado é transmitido também no caráter de transformação pessoal. Essa idealização do corpo ideal, além de ser inspirada por alguns ídolos, enalteceu mais uma vez a cobiça pelo corpo proporcional. É justamente essa ilusão de que existem partes desproporcionais em seu corpo, que o vigoréxico assume um dos sinais do transtorno dismórfico corporal.
As análises advindas deste estudo permitem sugerir forte indício de sintomas da dismorfia muscular como constatado por Olivardia (2001), nos quais os exercícios físicos foram responsáveis por grande parte das atividades do vigoréxico, que destina a vida à academia, se sentindo rejeitado, discriminado ou deprimido se ficar um dia sem exercício físico ou sem o uso de esteroides anabolizantes, chegando a comprometer as atividades sociais, ocupacionais, recreativas e até mesmo os relacionamentos interpessoais. Este comportamento foi possível ser observado no momento que um dos atores sociais chama a atenção:

"Terminei inclusive um namoro em razão disso, ela dizia que eu me preocupava mais com meu corpo do que com o que ela sentia... (Adônis)"

É fácil perceber que estas modificações sociais, principalmente no que concerne a prática exacerbada do treinamento de força, são incentivadas diante da expansão dos meios de comunicação, nos quais se tem enfatizado o culto à forma física e à boa aparência, o que contribui para um maior interesse e desejo por um corpo esteticamente perfeito (Carvalho, Rodrigues, Meyer, Lancha Jr, \& De Rose, 2003; Grieve, Truba, \& Bowersox, 2009; Iriart \& Andrade, 2002).

Além disso, o ideal muscular é reforçado pelos significados ou respeito frente aos conceitos dos outros, como da família, dos amigos e da mídia. Estas fontes comunicam expectativas sobre os benefícios do ganho muscular, e essa expectativa possui um papel importante na promoção do corpo musculoso como sendo o ideal (Smolak, Murnen, \& Thompson, 2005; Stanford \& McCabe, 2005).

Para Baudrillard (1970), as mudanças dos códigos sociais por meio das narrativas midiáticas se justapõem às experiências vividas, produzindo a realidade por meio de cópias e simulações. Dando sequência às denúncias que envolvem a indústria cultural e adicionando-se à crítica de Jameson (1996) referente à estetização da realidade, Baudrillard nomeou de 
"esquizofrenia cultural" o processo de invenção midiática do real. E vai além quando indica que o que ultimamente faria girar a roda do capitalismo, seriam "investimentos libidinais no imaginário". Acredita-se que estes autores afirmam que a onipresença da mídia determina uma repercussão de imagens que acaba por asfixiar o próprio registro do imaginário, ou seja, a possibilidade criativa do indivíduo que deseja desenhar uma estética para aquilo que anseia.

Tanto a obsessão quanto a compulsão pela prática exacerbada do treinamento de força em busca do corpo perfeito podem gerar outras consequências maléficas ao vigoréxico, como o overtraining. Este se caracteriza pelo excesso de treinamento, ou seja, tempo de recuperação incompleto entre as sessões de treinamento, provocando manifestações de ordens físicas, psíquicas ou emocionais, tais como perda de apetite e peso corporal, insônia, irritabilidade, depressão, ansiedade, agressividade, lesões, diminuição da libido, fraqueza, cansaço constante, dificuldade de concentração e dores músculo-articulares (Assunção, 2002; Fleck \& Kraemer, 2006).

Observou-se nos relatos características desses sintomas que associam a dismorfia muscular ao overtraining, como é possível observar nas falas:

"Insônia eu sempre tive [...]. O desinteresse sexual é comum dependendo das dosagens de ergogênicos misturadas, principalmente no caso de testosterona, pois durante o seu uso, é como se tivesse ingerindo várias doses de Viagra ao dia pois a libido vai nas alturas e algumas semanas após descontinuado seu uso, o quadro acaba revertendo devido a desaceleração da produção natural do hormônio pelo organismo devido as doses sintéticas que foram injetadas (2)."

"Já senti insônia, lesões musculares, sentimento de inferioridade, desmotivação (Adônis)."

"Senti insônia, fraqueza, lesões poucas vezes e desmotivação, porém nunca desisti, além de sentir enjôo quase todo treino (ultimamente), e tremedeira e palpitação poucas vezes (Rocky)."

"Já senti insônia, fraqueza, lesões musculares, sentimento de inferioridade e depressão (Minotauro)."

A ocorrência dessas características, principalmente o evento de lesões, pode ser uma das consequências do estado de overtraining ao qual o indivíduo se submete na busca pelo corpo ideal. Essa insistência em continuar com o treinamento mesmo após lesões pode estar significativamente associada aos sinais de dismorfia muscular. Além disso, Nagayama, Porta, Chaud, Marchioni e Ribeiro (2000) e, Calfee e Fadale (2006) sugerem que a falta de orientação adequada por meio dos profissionais de saúde pode estar permitindo parte da sociedade a usar substâncias que possam realizar seus desejos estéticos de maneira imediatista, como os EAA.

\section{Esteroides Anabólicos Androgénicos}

O uso e abuso dos EAA têm aumentado entre os praticantes do treinamento de força, principalmente entre os vigoréxicos (Ferreira, Ferreira, Azevedo, Medeiros, \& Silva, 2007; Silva, Danielski, \& Czepielewski, 2002). Neste sentido, dados advindos deste estudo constataram que $35 \%$ dos participantes masculinos e $5 \%$ dos femininos relataram utilizar pelo menos uma das seguintes substâncias: nandrolona, testosterona, stanozolol, clembuterol, oximentolona, durateston, deca durabolin, oxandrolona, pulmonil, deposteron, Primobolan, dexa, propionato de testosterona, trembolona, boldenona, efedrina, turinabol e lipostabil ${ }^{\circledR}$. Sobre este ponto, destacase o discurso de Rambo:

"Já fiz uso de ergogênicos. De início foram uma opção para tratamento de lesão que me poderia trazer benefícios musculares também, então, comecei com o uso de nandrolona acompanhada de um mix de testosterona. Anos mais tarde acabei testando também stanozolol, clembuterol e oximentolona." 
Sob este aspeto, o uso de esteroides anabolizantes torna ainda mais nítida a busca irracional pelo corpo musculoso, sendo irrelevante as consequências desta busca, sejam elas psicológicas, fisiológicas e/ou sociais. Estudos recentes de Kanayama, Brower, Wood, Hudson e Pope Jr (2009) e Rohman (2009) mostraram que os EAA estão associados à predisposição, precipitação ou perpetuação dos fatores psiquiátricos da dismorfia muscular que incluem complicações como alterações de humor e comportamento, anormalidades na percepção corporal e sinto-mas de abstinência. Assim, o constante uso e abuso dos EAA podem ocorrer em virtude da dismorfia muscular com o objetivo de alcançar um corpo cada vez mais musculoso em um pequeno intervalo de tempo.

"Hoje em dia penso em usar algo que me ajude pra ter o corpo perfeito (Belfort)."

Brower (1992), e Middleman e Durant (1996) revelam que ocorrem síndromes de abstinência provocadas pela dependência e interrupção do uso de EAA, sendo consistentes com os critérios da dismorfia muscular e têm o potencial de desencadear crises comportamentais, como as decorrentes deste transtorno. Silva et al. (2002) demonstram que tais efeitos comportamentais podem estar relacionados ao uso de metandiona, devido à alteração da função serotoninérgica causada por esta substância. Estas síndromes aparecem em cerca de $14 \%$ a $57 \%$ dos casos (Brower, 1992), nos quais, segundo Wroblewska (1996), percebemse sintomas como perda de controle, depressão, fadiga, inquietação, perda de apetite, insônia, decréscimo da libido e dores de cabeça.

Assunção (2002), Gomes (2005), e Pope Jr e Katz (1994) tem relatado alterações de imagem corporal entre levantadores de peso e fisiculturistas, principalmente entre os que utilizam EAA. De uma maneira geral, os resultados de pesquisas convergem para o fato de que os levantadores de peso portadores de dismorfia muscular utilizam anabolizantes com maior frequência e em maior quantidade, quando comparados àqueles que não apresentam os sintomas da vigorexia. É válido ressaltar que, dentre os vigoréxicos que apresentam os sintomas, os usuários de anabolizantes demonstram quadro mais grave (Assunção, 2002).

Em 1993, Pope Jr, Katz e Hudson analisando uma amostra de 108 fisiculturistas (com e sem uso de esteroides anabolizantes), relataram o que foi denominado na época de anorexia nervosa reversa. Nesta amostra, foram identificados nove indivíduos (8.3\%) que se descreviam como muito fracos e pequenos, quando na verdade eram extremamente fortes e musculosos. Ademais, todos mencionavam uso de EAA e dois tinham história anterior de anorexia nervosa. Demonstrou-se ainda que quatro entre nove fisiculturistas com dismorfia muscular desenvolveram o transtorno somente depois que começaram a fazer uso de esteróides anabolizantes, o que indica que estas drogas facilitam, em muitos casos, a distorção da imagem corporal. Além disso, segundo Lise, Gama e Silva, Ferigolo e Barros (1999), a dismorfia muscular acarreta outros problemas físicos e estéticos podendo estar relacionado ao uso de EAA, como por exemplo, a desproporção displásica, caracterizada pela desproporção entre segmentos corporais e problemas ósseoarticulares causados tanto pelo peso corporal excessivo, quanto pela sobrecarga exagerada durante o exercício.

Os problemas graves mais percebidos atualmente são a adesão às drogas nas farmácias, na mídia eletrônica e sua crescente popularização entre as pessoas que frequentam academias de ginástica, principalmente entre os vigoréxicos, além dos efeitos colaterais letais decorrentes de seu uso abusivo.

\section{Aspetos Psicossociais}

Os achados do presente estudo corroboram com a descrição da dismorfia muscular. Esta deve ser considerada como um transtorno da linhagem obsessivo-compulsiva, tanto pela obsessão em musculatura, quanto pela compulsão aos exercícios. Ainda, é constante a distorção do esquema corporal e a ingestão de 
recursos ergogênicos (Gomes, 2005; Pope Jr \& Katz, 1994; Torres et al., 2005). Em relação à perceção corporal, novamente pode-se observar que tanto os homens quanto as mulheres descreveram relatos similares aos encontrados na literatura, ou seja, $80 \%$ dos atores sociais se consideram magros/fracos, não se consideram fortes e musculosos mesmo descrevendo um grande volume muscular como relatado a seguir. Este fato pode conduzir a alterações na perceção do esquema corporal, como observado nestes recortes:

"Apesar de braços de $46 \mathrm{~cm}$ frio (sem estar aquecido durante o treinamento) acho eles finos (Hulk)."

"Me olho no espelho e sempre me acho fraco $e$ magro, mesmo estando com $11 \%$ de gordura, 90 quilos e um metro e oitenta e cinco de altura (Conan)."

Assim, os resultados sugerem que os atores apresentam traços característicos da dismorfia muscular sugeridos por Pope Jr e Katz (1994), nos quais os portadores demonstram alterações quanto à perceção da real imagem corporal de si, bem como alterações na personalidade e comportamentos, como baixa autoestima, dificuldades para integrar-se socialmente, timidez e possa, frequentemente, rejeitar ou aceitar com sofrimento a própria imagem corporal, não se considerando forte o suficiente ao acreditar que sempre está faltando "algo a mais" em sua musculatura. Facilmente essa insatisfação pôde ser verificada:

\section{"Nunca estou forte o suficiente (Adônis)." \\ "Acho que sempre falta alguma coisa (Super Homem)."}

"Me sinto sempre fraco, acho que pego pouco peso, mesmo todos dizendo que não (Rocky)."

Notadamente há uma insatisfação com o próprio corpo. Neste caso, ressalta-se o fato de ser comum, mesmo quando se está com o somatotipo adequado, sentirem-se com a imagem corporal inadequada, apresentando, desta maneira, alguma alteração na perceção da autoimagem. Esta alteração pode ser resultado das pressões exercidas sobre o sujeito, principalmente pelo contexto sociocultural no qual o indivíduo está situado e pela mídia. No entanto, por meio das experiências vividas o sujeito constrói uma visão intimamente associada do eu e da imagem corporal, ambos dependentes do desenvolvimento do sistema sensorial, neurológico, da perceção e das relações sociais e culturais, o que permite ao indivíduo escapar da passividade frente às pressões e, intervir de modo autônomo, no sistema das relações sociais como detentor de suas decisões e de suas ações.

Outro traço psicológico comum à dismorfia muscular, e que foi claramente narrado pelos participantes da pesquisa, refere-se à constante checagem dos ganhos musculares, com repetidas observações de seu corpo no espelho. Não surpreendentemente, $90 \%$ dos participantes ( $75 \%$ dos homens e $100 \%$ das mulheres) afirmaram esta prática compulsiva. A seguir, algumas falas:

"Sim, mais ou menos umas 40 vezes por dia me olho no espelho. Antes do treino e depois (Conan)."

"Sim, constantemente (Mulher Maravilha, She-ra, Rambo, Hulk, Coleman, Super Homem)."

"Sim, eu às vezes me acho narcizista (Rocky)."

De acordo com Olivardia (2001), a obsessão da beleza física se converte em algumas doenças emocionais como ansiedade, depressão, fobias, atitudes compulsivas e repetitivas como seguidas observações no espelho, possíveis ganhos de massa muscular checados até 13 vezes ao dia, e que conduzem à dismorfia muscular, estando relacionada com uma patologia psíquica das pessoas excessivamente preocupadas com a aparência, não estando satisfeitas 
com seus músculos e sempre estão na busca obsessiva pela perfeição, como pode ser observado no relato de Conan:

"Eu quando fico sem treinar um tempo, sinto dores musculares e depressão por que penso que estou ficando fraco, magro e com isso eu perco a vontade de me alimentar direito."

Os comportamentos compulsivos são hábitos apreendidos e seguidos por alguma gratificação emocional, normalmente um alívio de ansiedade e/ou angustia, acontecendo quase automaticamente, sendo executadas várias vezes (Gomes, 2005; Pope Jr \& Katz, 1994).

Quanto aos benefícios em ser musculoso, ambos os gêneros descreveram as mesmas respostas, nas quais $50 \%$ dos participantes relataram aumento da autoestima, 35\% apresentaram melhoras no aspeto social, principalmente ao afirmarem que o corpo musculoso "impõe respeito" sendo mais aceitos em seus grupos sociais e $15 \%$ melhorias estéticas, características que são confirmadas pela literatura científica (Gomes, 2005; Silva \& Moraes, 2006). De acordo com Olivardia (2001) a preocupação excessiva com a imagem corporal geralmente provoca insegurança social, podendo agravar uma introversão existente. A atitude comum é acreditar que a timidez e a insegurança social seriam resolvidas tendo corpos belos e fortes.

\section{Imagem Corporal}

Quanto à aparência, $85 \%$ dos sujeitos $(70 \%$ dos homens e $100 \%$ das mulheres) relataram a hipertrofia muscular como objetivo ao praticar o treinamento de força, idealizando um corpo musculoso, proporcional e com baixo percentual de gordura. Assim, está claro que os objetivos estéticos são os mais desejados pelos praticantes dessa modalidade de exercício físico, apresentando traços semelhantes aos sintomas psicossociais da dismorfia muscular.

Achados de Santos e Santos (2002) observaram que $69 \%$ dos frequentadores de academia de ginástica possuíam objetivos de hiper- trofia. Em Goiânia (GO), estudo realizado em 15 academias, onde 99\% dos entrevistados praticavam exercício de força, foi detetado que $60.5 \%$ deles desejavam aumentar a musculatura corporal (Silva \& Moraes, 2006). Pope Jr, Phillips e Olivardia no ano de 2000 descobriram que, em média, os homens gostariam de ter um corpo com 12.7 quilos a mais de músculos. Isto torna evidente a participação de fatores socioculturais na etiologia da dismorfia muscular, sendo intermediados pela hipervalorização da imagem musculosa.

Considerando a importância da compreensão dos participantes do estudo sobre a valorização da imagem corporal hiper musculosa, foi indagado se algum aspeto na aparência física os incomodava a ponto de deixá-los deprimidos, ansiosos ou zangados com isso. Com esta questão buscou-se identificar possíveis insatisfações corporais um dos sinais principais da dismorfia muscular. Sob este olhar, $85 \%$ dos atores $(70 \%$ dos homens e $100 \%$ das mulheres) responderam que sim, como ilustrado a seguir.

"Às vezes acho que meus ganhos cessaram e fico nervoso com isso (He-Man)."

"Às vezes em dado período de ganha ou perda de volume e/ou definição, o atleta acaba passando por modificações que pode fazer com que a motivação psicológica melhore ou piore de acordo com os resultados. Isso é normal [...] Mas, ficar deprimido faz parte do processo às vezes ou inconformado, é justamente essa cobrança que ajuda a caminhar no caminho da melhora e da superação (Rambo)."

A partir destas falas importa evidenciar a influência da dimensão sociocultural na imagem corporal, propagando um ideal de saúde em razão do consumo de inúmeros produtos, utilizando como isca um modelo específico de estética corporal. Conforme as palavras de Anzai (2000, p. 73): "...Se a televisão, a publicidade, o cinema, as revistas, os jornais, e agora a internet, defendem as dietas milagrosas, os músculos tor- 
neados e bronzeados, as vitaminas que evitam o envelhecimento, as clínicas de rejuvenescimento e as academias de ginástica, é porque isso tudo dá muito dinheiro. E se muito pouco se fala de afeto e respeito entre as pessoas comuns, não tão lindas e nem tão elegantes como as modelos, mas, que mesmo assim, se sentem felizes, certamente é porque isso é bem menos rentável".

Assim, Galli e Reel (2009) em estudo de evidência qualitativa afirmaram que esta influência foi citada por $80 \%$ dos praticantes de treinamento de força, emergindo quatro categorias: religião, relacionamento com o sexo oposto, os meios de comunicação e, comentários e expectativas a cerca de seus corpos. No presente estudo, é notória a explicação de duas das quatro categorias supracitadas, sendo elas, relacionamento com o sexo oposto e expectativas sobre sua imagem corporal:

"Quanto à aparência de musculoso, além de ser ótimo para autoestima, pode ser vantajoso com o sexo oposto. Fora isso me foi oferecida algumas oportunidades como desfiles, patrocínio e de modo geral uma certa popularidade que me pode ser benéfica no meu ramo profissional de atuação da área jurídica e de vendas (Rambo)."

Estes resultados demonstraram que constantemente esses indivíduos sentem-se insatisfeitos com a imagem corporal adquirida, apresentando compulsão em praticar exercícios com pesos para minimizarem tais sentimentos e alcançarem os corpos hipertrofiados como desejam.

\section{Aspetos Nutricionais}

Além da obsessão pelo corpo perfeito, a dismorfia muscular produz uma importante mudança na rotina e costumes dos indivíduos, notadamente na questão alimentar, ocorrendo modificações radicais na dieta, que passa a ser hiperproteica e acompanhada de diversos suplementos alimentares, principalmente daqueles à base de aminoácidos e proteínas (Olivardia, 2001). Nesse sentido, a presente pesquisa vem corroborar com a literatura visto que homens e mulheres (90\% dos participantes) se preocupam e modificam, indistintamente, a alimentação. Esta passa a ser hiperproteica (40\%) ou com restrição lipídica (25\%). E ainda, hiperproteica e hipolipídica (25\%). Assim, foram obtidos os seguintes relatos:

$$
\begin{aligned}
& \text { "Preocupação em alto consumo de proteínas } \\
& \text { (Apolo)." }
\end{aligned}
$$

"Busco sempre uma alimentação balanceada com pouca gordura e sempre com níveis proteicos elevados (Adônis)."

"Tenho minha dieta montada, me alimento de duas em duas horas, procuro comer proteínas de qualidade, gorduras boas e carboidratos de baixo indice glicêmico. Faço oito refeições diárias (He-Man)."

Ainda no tocante a alimentação, $90 \%$ dos indivíduos (80\% dos homens e $66.6 \%$ das mulheres) relataram consumir suplementos alimentares, em especial os hiperproteicos e os compostos por aminoácidos. Segundo Ferreira et al. (2007), devido à óbvia associação entre as proteínas e os músculos, além da quantidade significativa de informações equivocadas proveniente de atletas e comerciantes acerca das virtudes de dietas ricas em proteínas e do grande apelo mercadológico, estes nutrientes podem estar sendo consumidos em excesso, provocando prejuízos à saúde e desperdício financeiro, uma vez que os suplementos possuem custo relativamente alto. Na maioria dos casos os consumidores de suplementos, incluindo os vigoréxicos, não são orientados e acompanhados por profissionais habilitados, sendo mais comuns indicações variadas ou a simples procura em meios eletrônicos ou em lojas de suplementos.

Evidenciou-se também que o ambiente digital vem sendo utilizado para obtenção de informações e compra direta de suplementos alimentares, prática que indubitavelmente estimula o consumo indiscriminado destes produtos. 


\section{Limitações e direções futuras}

Não obstante estes resultados forneçam informações significativas sobre as experiências da dismorfia muscular, uma limitação deste estudo foi a baixa resposta dos atores participantes, visto que, 125003 praticantes de treinamento de força tiveram a opção de receber o questionário enviado pelos pesquisadores, mas, apenas 20 atores sociais responderam e compuseram o grupo estudado. Este fato pode ter ocorrido por receio dos sujeitos em ser identificados uma vez que suas inserções na mídia eletrônica fornecem informações pessoais com riqueza de detalhes, podendo ser visualizado por qualquer outro integrante do site.

Os resultados deste estudo reúnem informações substanciais para se refletir a saúde dos indivíduos que apresenta compulsão pelo treinamento de força. Portanto, investigações futuras sobre a dismorfia muscular estão sendo desenvolvidas no Laboratório de Estudo e Pesquisa em Lazer, Esporte, Corpo e Sociedade LAECOS da Universidade Federal da Paraíba, Brasil, para corroborar com os dados analisados, examinando o papel sociocultural que envolve a dismorfia muscular associada à imagem corporal, ao treinamento de força e uso de recursos ergogênicos.

\section{CONCLUSÕES}

Com base nas análises sobre a dismorfia muscular foi possível inferir que é uma temática pouco estudada, prevalente em indivíduos do sexo masculino, principalmente entre indivíduos praticantes do treinamento de força que buscam uma aparência física hegemônica por meio de um corpo musculoso. O transtorno pode ter sua origem parcialmente explicada por fatores psicossociais, relacionados a uma crescente pressão exercida principalmente pela mídia para que os indivíduos tenham corpos fortes e musculosos motivando um aumento na incidência da DM.

Tanto homens quanto mulheres com dismorfia muscular percebem o estado de insatisfação corporal, anseiam pelo corpo ideal e dificilmente reconhecem limites físicos e psicológicos para a aquisição de um corpo hiper musculoso. Estas insatisfações, por sua vez, comummente acarretam preocupações e interferências nas atividades pessoais e profissionais dos sujeitos, embora aceitem sua aparência física com algum sofrimento. A dismorfia muscular está associada ao sofrimento e prejuízos em diversas áreas da vida das pessoas. Além disso, salienta-se que homens e mulheres apresentam os mesmos desejos e comportamentos diante do anseio pela conquista, a qualquer custo, do corpo ideal e musculoso. Adicionalmente, como demonstrado, sua presença pode aumentar o risco de uso dos esteroides anabolizantes, drogas com consequências potencialmente perigosas, bem como, de suplementos alimentares de forma indiscriminada, sem acompanhamento de um profissional especializado.

Este estudo teve a preocupação com a hipervalorização e incentivo do culto ao corpo, procurando preencher algumas das lacunas relacionadas ao conhecimento sobre a dismorfia muscular. Espera-se ainda oferecer subsídios para a tomada de consciência da necessidade de haver um trabalho multiprofissional apropriado para o alcance de resultados significativos na prevenção e tratamento da dismorfia muscular.

Agradecimentos:

Nada a declarar.

Conflito de Interesses:

Nada a declarar.

Financiamento:

Nada a declarar.

\section{REFERÊNCIAS}

Amatuzzi, M. (1993). Etapas do processo terapêutico: Um estudo exploratório. Psicologia: Teoria e Pesquisa, 9, 1-21.

Anzai, K. (2000). O corpo enquanto objeto de consumo. Revista Brasileira de Ciências do Esporte, 21(2), 71-76. 
Araújo, A., \& Soares, Y. (1999). Perfil de utilização de repositores protéicos nas academias de Bélem, Pará. Revista de Nutrição, 12 (1), 5-19.

Arraz, C. H. (2010). Jornal correio brasiliense. Consultado em 15 de abril de 2010 a partir de http:// www.correiobraziliense.com.br

Assunção, S. S. (2002). Muscle dysmorphia. Revista Brasileira de Psiquiatria, 24, 80-84.

Azevedo, A. (2008). Substâncias injetáveis não anabolizantes na prática da musculação: Uso e riscos (Monografia de graduação não publicada). Departamento de Educação Física da Universidade Federal da Paraíba, Brasil.

Bardin, L. (2002). Análise de conteúdo. Lisboa: Ed. 70.

Baudrillard, J. (1970). A sociedade de consumo. São Paulo: Martins Fontes.

Beiras, A., Lodetti, A., Cabral. A. G., Toneli, M. J., \& Raimundo, P. (2007). Gênero e super-heróis: O traçado do corpo masculino pela norma. Psicologia \& Sociedade, 19(3), 62-67.

Brower, K. J. (1992). Clinical assessment and treatment of anabolic steroid users. Psychiatric Annals, 22, 3540-3544.

Calfee, R., \& Fadale, P. (2006). Popular ergogenic drugs and supplements in young athletes. Pediatrics, 117, 577-589.

Carvalho, T., Rodrigues, T., Meyer, F., Lancha Jr, A. H., \& De Rose, E. H. (2003). Modificações dietéticas, reposição hídrica, suplementos alimentares e drogas: Comprovação de ação ergogênica e potenciais riscos para a saúde. Revista Brasileira de Medicina do Esporte, 9(2), 43-56.

Cohane, G. H., \& Pope Jr, H. G. (2001). Body image in boys: A review of literature. International Journal of Eating Disorders, 29, 373-379.

Dawes, J., \& Mankin, T.(2004). Muscle dysmorphia. Strenght and Conditioning Journal, 26, 24-25.

Fairburn, C. (1994). Eating disorders. In R. Kendell \& A. K. Zeally (Eds.), Companion to psychiatric studies (pp. 525-542). London: Butler \& Tanner.

Ferreira, U. M., Ferreira, A. C., Azevedo, A. M., Medeiros, R. L., \& Silva, C. A. (2007). Esteróides anabólicos androgênicos, Revista Brasileira em Promoção da Saúde, 20(4), 267-275.

Flack, S. J., \& Kraemer, W. J. (2006). Fundamentos do treinamento de força muscular. RS: Artmed.

Freitas, C. M. (2000). O significado social do desporto nas classes sociais: Uma análise do fenômeno (Tese de doutorado não publicada). Universidade do Porto, Portugal.

Frizon, F., Macedo, S. M., \& Yonamine, M. (2005). Uso de esteróides andrógenos anabólicos por praticantes de atividade física das principais academias de Erechim e Passo Fundo/RS. Revista de Ciências Farmacêuticas Básica e Aplicada, 26(3), 227-232.

Galli, N., \& Rell, J. J (2009). Adonis or Hephaestus? Exploring body image in male athletes. Psychology of Men \& Masculinity, 10(2), 95-108.

Gomes, M. V. (2005). Vigorexia: Colocando a vaidade acima da saúde mental (Monografia de graduação não publicada). CESMAC, Brasil.

Grieve, F. G. (2007). A conceptual model of factors contributing to the development of muscle dysmorphia. Eating Disorders, 15, 63-80.

Grieve, F. G., Truba, N., \& Bowersox, S. (2009). Etiology, assessment, and treatment of muscle dysmorphia. Journal of Cognitive Psychotherapy: An International Quarter, 23(4), 306-314. doi: 10. 1891/0889-8391.23.4.306

Hildelbrandt, T., Langenbucher, J., \& Schlundt, D. G. (2004). Muscularity concerns among men: Development of attitudinal and perceptual measures. Body Image, 1, 169-181. doi: 10.1016/ j.bodyim.2004.01.001

Iriart, J. A., \& Andrade, T. M. (2002). Musculação, uso de esteróides anabolizantes e percepção de risco entre jovens fisiculturistas de um bairro popular de Salvador, Bahia, Brasil. Cadernos de Saúde Pública, 18(5), 1379-1387.

Jameson, F. (1996). Pós-modernismo: A lógica cultural do capitalismo tardio. São Paulo: Ática.

Kanayama, G., Brower, K. J., Wood, R. I., Hudson, J. I., \& Pope Jr, H. (2009). Anabolic-androgenic steroid dependence: An emerging disorder. Addiction, 104, 1966-1978. doi: 10.1111/j.13600443.2009.02734.x

Lantz, C. D., Rhea, D. J., \& Mayhew, J. L. (2001). The drive for size: A psycho-behavioral model of muscle dysmorphia. International Sports Journal, 5, 71-86.

Lise, M. L., Gama e Silva, T. S., Ferigolo, M., \& Barros, H. M. (1999). O abuso de esteróides anabólico-androgênicos em atletismo. Revista da Associação Medica Brasileira, 45(4), 364-370.

Lollo, P., \& Tavares, M. (2004). Perfil dos consumidores de suplementos dietéticos nas academias de ginástica de Campinas. Lecturas Educación Física y Deportes, 10(76).

Middleman, A. B., \& Durant, R. H. (1996). Anabolic steroid use and associated health risk behaviours. Sports Medicine, 21, 251-255.

Nagayama, C., Porta, D., Chaud, D. M., Marchioni, D. M., \& Ribeiro, S. M. (2000). Avaliação do 
conhecimento de estudantes de educação física quanto à nutrição e orientação nutricional. Revista Nutrição em Pauta, 4, 12-16.

Olivardia, D. M., Pope Jr, H. G., \& Hudson J. I. (2000). Muscle dysmorphia in male weightlifters: A case control study. American Journal of Psychiatry, 157(8), 1291-1296.

Olivardia, R. (2001). Mirror, morror on the wall, who's the largest of them all? The features and phenomenology of muscle dysmorphia. Harvard Revision Psychiatry, 9(5), 254-259.

Pereira, D. A., Doimo, L. A., \& Kowalski, M. (2009). Discurso estético do corpo: O auto cuidado de mulheres. Revista Eletrônica de Ciências Sociais, 3(6), 33-59.

Pereira, R., Lajolo, F., \& Hirschbruch, M. (2003). Supplement consumption among fitness center users in São Paulo, Brazil. Revista de Nutrição, 16(3), 265-272. doi: 10.1590/S1415-52732003 000300004

Pope Jr, H. G., \& Katz, D. L. (1994). Psychiatric and medical effects of anabolic-androgenic steroid use. Archives of General Psychiatry, 51, 375-382.

Pope Jr, H. G., Gruber, A. J., Choi, O., Olivardia, R., \& Phillips, K. A. (1997). Muscle dysmorphia: An underrecognized form of body dysmorphic disorder. Psychosomatics, 38, 547-548.

Pope Jr, H. G., Phillips, K., \& Olivardia, R. (2000). The Adonis complex: The secret crisis of male body obsession. New York: Free Press.

Pope Jr, H.G., Katz, D. L., \& Hudson, J. L. (1993). Anorexia nervosa and "reverse anorexia" among 108 male bodybuilders. Comprehensive Psychiatry, 34, 406-409.

Reis, M., Manzoni, M., \& Simonard-Loureiro, H. (2006). Avaliação do uso de suplementos nutricionais por freqüentadores de academias de ginástica em Curitiba. Revista de Nutrição, 5(5), 56-61.

Rohman, L. (2009). The relationship between anabolic androgenic steroids and muscle dysmorphia: A review. Eating Disorders, 17, 187-199.

Santos, M. A., \& Santos, R. P. (2002). Uso de suplementos alimentares como forma de melhorar a performance nos programas de atividade física em academias de ginástica. Revista Paulista de Educação Física, 16, 174-185.
Silva Júnior, S. H., Souza, I., Silva, J. H., Oliveira, J. W., \& Souza, M. A. (2008). Perfil de atletas de academia: O uso de anabolizantes e suplementos nos programas de atividade física. Lecturas Educación Física y Deportes, 13(119).

Silva, L. S., \& Moreau, R. L. (2003). Uso de esteróides anabólicos androgênicos por praticantes de musculação de grandes academias da cidade de São Paulo. Revista Brasileira de Ciências Farmacêuticas, 39(3), 327-333.

Silva, M. S., \& Moraes, A. S. (2006). Utilização de suplementos alimentares por indivíduos que praticam exercícios físicos em academias de Goiânia. IX Semana Científica da FEF/UFG, Goiânia, Brasil.

Silva, P. R., Danielski, R., \& Czepielewski, M. A. (2002). Anabolic steroids in sports. Revista Brasileira de Medicina do Esporte, 8, 235-243. doi: 10.1590/S1517-86922002000600005

Silva, P. R., Machado Júnior, L. C., Figueiredo, V. C., Cioffi, A., Prestes, M. C., \& Czepieliwski, M. A. (2007). Prevalência do uso de agentes anabólicos em praticantes de musculação de Porto Alegre. Arquivos Brasileiros de Endocrinologia $e$ Metabologia, 51 (1), 104-110.

Smolak, L., Murnen, S., \& Thompson, J. K. (2005). Sociocultural influences and muscle building in adolescent boys. Psychology of Men and Muscularity, 6, 227-239.

Stanford, J. N., \& McCabe, M. P. (2005). Sociocultural influences on adolescent boys body image and body change strategies. Body Image, 2, 105113. doi: 10.1016/j.bodyim.2005.03.002

Swami, V., \& Tovvé, M. J. (2007). Adult attachment and body satisfaction an exploration of general and specific relationship differences. Body Image, 4, 391-396.

Torres, A. R., Ferrão, Y. A., \& Miguel, E. C. (2005). Body dysmorphic disorder: An alternative expression of obsessive-compulsive disorder? Revista Brasileira de Psiquiatria, 27, 95-96.

Vieira, J. L., Rocha, P. G., \& Ferrarezzi, R. (2010). A dependência pela prática de exercícios físicos e o uso de recursos ergogênicos. Acta Scientiarum. Health Sciences, 32 (1), 35-41.

Wroblewska, A. M. (1996). Androgenic-anabolic steroids and body dysmorphia in young men. Journal of Psychosomatic Research, 42, 225-234.

(cc) EY-No Todo o conteúdo da revista Motricidade está licenciado sob a Creative Commons, exceto quando especificado em contrário e nos conteúdos retirados de outras fontes bibliográficas. 